\title{
Justice, equality and liberty: Inspiration from the Indian Constitution for effective diabetes management
}

\author{
SANJAY KALRA, MADHUR VERMA
}

\begin{abstract}
Diabetes Mellitus (DM) could have easily been labelled as the "black swan" of the 21st century, had Covid-19 not emerged as a pandemic. Modern diabetes care in India grapples with multiple challenges. The twin enemies of uncontrolled hyperglycaemia and unwanted hypoglycaemia pose an ethical dilemma during the decision-making process of DM management. With an unfavourable support system against this rapidly emerging public health threat, we look to the Indian Constitution for guidance. Apart from just euglycaemia (a state of normal blood glucose levels), every Indian living with DM has some requirements and rights, that can be summarised with the help of three basic principles of the Constitution, ie, justice, equality and liberty. Together, these words remind us to practise accurate and appropriate diabetes care, delivered in a patient-centred manner. Justice, equality and liberty should be incorporated into diabetes-care systems, and fraternity encouraged. This will ensure that we achieve the dreams of our founding fathers through evolution of a comprehensive disease management model.
\end{abstract}

Keywords: Equality, ethical dilemma, Euglycaemia, justice, liberty, patient-centred-care.

\section{Background}

Contrary to the popular perception of non-communicable diseases (NCD) as diseases of the upper strata of the community, they disproportionately affect the marginalised sections in almost every part of the world, but substantially more in the low and middle-income countries (LMIC)(1). Amongst NCDs, diabetes needs no special mention. Indeed,

Authors: Sanjay Kalra (corresponding author - brideknl@gmail.com) Consultant Endocrinology, President, Endocrine Society of India, Bharti Hospital \& B.R.I.D.E., India, Karnal 132001 INDIA; Madhur Verma (drmadhurverma@gmail.com), Assistant Professor of Community and Family Medicine, All India Institute of Medical Sciences, Bathinda, Punjab, India. 151001 INDIA.

To cite: Kalra S, Verma M. Justice, equality and liberty: Inspiration from the Indian constitution for effective diabetes management. Indian J Med Ethics. 2021 Jul-Sep; 6(3) NS: 242-245 DOI: 10.20529/IJME.2021.012.

Published online first on February 23, 2021.

Manuscript Editor: Rakhi Ghoshal

Peer reviewer:Vijayaprasad Gopichandran

(c) Indian Journal of Medical Ethics 2021 diabetes is affecting (age-standardised prevalence) up to $8.5 \%$ of the global adult population, and a majority of them hail from LMICs (2). In India, the prevalence of diabetes in adults aged 20 years or older was estimated to be $7.7 \%$ (6.98.4) in 2016, with significant regional variations (3). Untreated Type 2 diabetes mellitus (DM) deteriorates the quality of life and substantially contributes to premature deaths. Detection in the early stages of the disease, lifelong treatment, along with lifestyle modifications, helps in decreasing mortality and morbidity.

However, modern diabetes care in India grapples with multiple challenges. The huge burden of undiagnosed and untreated disease coexists with over-labelled and mismanaged cases (4). Further, the use of suboptimal therapeutic regimens and preparations creates a paradoxical situation, where the physician constantly struggles to manage the twin enemies of uncontrolled and unwanted high or low blood glucose levels. These factors also pose an ethical dilemma during the decision-making process of DM management. The ineffective management of DM as a result of non-adherence to the prescribed treatment by patients, and therapeutic inertia amongst physicians are some of the major hurdles to achievement of the desired glycaemic targets. This eventually leads to suboptimal blood-sugar levels and a plethora of complications (5). In less developed nations with high levels of illiteracy, it is difficult to make the community understand the implications of chronic diseases such as DM, and increase compliance to drug adherence. Anecdotally, unqualified medical practitioners take advantage of this lack of confidence in allopathic treatment, and tend to get more favourable media patronage, in spite of the sheer lack of evidence in support of their claims. Unfounded hearsay is worsened by unregulated advertisements of unproven therapies and strategies for management of diabetes. The community at large tends to accept medical therapy from unregistered practitioners, on being assured of it as the gold standard in safety and tolerability. When untrained/unregistered practitioners casually market the concept of comprehensive wellness, banking on the internalisation by the public of the biopsychosocial construct of health, they eventually dilute the concept of mind-body medicine (6). Further, physicians have failed to convince governments that chronic diseases like 
DM represent a serious potential roadblock in the way towards universal health care in India. Unlike communicable diseases like Covid-19, the actual effects of NCDs may not appear as a national emergency. These diseases are "lifestyle diseases", and are actually driven by corporate giants and national health policies. Products like cola, chips and pizzas have been so much assimilated into our lives that it is near impossible to drive them out without concerted government support.

In such a difficult scenario, we look to the Indian Constitution for guidance. Said to be the longest constitution in the world, the Constitution of India sets out the guiding principles for the governance of India. With 395 Articles divided into 22 parts, it is the supreme authority of the country. The three values that the constitution assures its citizens- justice, equality and liberty-should define our work as well. However, the Constitution of India does not expressly guarantee a fundamental right to health, but there are multiple references in the Constitution to public health. The apex court has reaffirmed that the right to health is fundamental and is interpreted to the right to life and personal liberty under Article 21. It is on record that the government has a constitutional obligation to provide health services (7). Within this obligation is the "right to diabetes care" and that too in an affordable and appropriate manner. As responsible health-care professionals, we demand the right to euglycaemia for every Indian as a noble target. Euglycaemia is not just normal blood glucose level, but a holistic diabetes care model, that has its roots in efforts at individual, and community level, with enabling environmental and social determinants which ensure self-care, and effective adoption of appropriate life-style changes. Therefore, apart from just normal blood glucose levels, as usually envisaged for every Indian living with DM, there are some more requirements, and rights, that can be summarised with the help of three basic principles of the Constitution:

\section{Justice}

Justice, in the diabetes care scenario, would imply offering the correct screening, diagnostic, monitoring, and therapeutic tools to all those who are in need (8). However, a growing literature links DM not to the individual but to "the web of the social determinants of health as it influences the quality of early life, stress, availability and quality of food and social exclusion", and hence, diabetes management should be envisaged under the holistic care model (9). Diabetes management can be unpleasant for many because of the numerous obligations (healthy eating habits, adequate physical activity and medication adherence); when compounded with food insecurity, this becomes an even greater barrier to adequate care (10). Food-insecure populations are at risk for monotonous, lower quality food, decreased micronutrient intake and associated deficiency disorders, and lesser servings of fruits and vegetables per week (11).This emphasises what it takes to ensure that everyone has the access to the means to manage the disease and its complications. We mentioned earlier, DM is a lifestyle disease. Factors like sedentary lifestyle, increased body weight, unhealthy diet, and substance abuse, are equally common, or may be more among the marginalised sections of the society. These inequalities call for a major public-policy overhaul. For example, the policies of promoting rice and wheat through the public distribution system without a focus on other nutrients, and inaccessibility to parks and play-areas for schools meant for low-socio economic strata, and their impact on the diabetes status of the population demands justification.

Prevention programmes that promote a wholesome dietary intake and physical activity can decrease the prevalence of DM and minimise the associated morbidity. Accessibility and affordability (NCDs receive only $1 \%$ of global health funding in $\mathrm{LMIC}$ ) to the essential medicines and technologies for DM is also a challenge in LMIC, compared to the developed world (12). Specifically, India spends around $0.5 \%$ of its GDP on NCDs and injuries and the gap between this frugal spending and Disability Adjusted Life Years (DALY) affects the economically vulnerable states most severely (13). The scientific community has appropriately labelled this disparity as the "social justice issue of our time." There is an urgent need to change this trend, or else any gains made from improvements in global health in the last few decades will fade away. These failings typify a major chunk of DM cases in LMIC which are undiagnosed or inadequately managed.

Therefore, the health administration and other stakeholders must admit to the disproportionate impact of DM on their vulnerable populations, and commit more support in terms of attention and financing to cope with the increasing burden of such chronic diseases. Or else, this can have significant human and economic consequences. Deficiencies in the accessibility to affordable treatment must be addressed, with the help of better logistical forecasting, distribution systems, justifiable pricing, and capping if necessary. Moreover, research and development of low-cost DM management tools that are suited for LMIC must be encouraged. A Common platform for different stakeholders to promote inter-disciplinary collaborations can create effective, affordable, and appropriate solutions that are the need of the hour. Appropriate justice should include penalisation of the culprits as well. Therefore, increased taxation on tobacco-products and junk foods should be implemented, so that the raised prices can restrain consumers from unstoppable consumption (14). Also, cities and workplaces should be organised to promote a healthy lifestyle and the International Diabetes Federation's (IDF) "diabetes prevention score for cities" can be seen as a guiding light for all (15).

\section{Equality}

Equality means that all persons seeking healthcare should be treated equally, without discrimination on any grounds. The Sustainable Development Goals (objective SDG 3.4) 
commits to reduce the NCD associated premature mortality by one-third by 2030, and promote mental-health and wellbeing (16). But, access to adequate treatment and management of DM is unfortunately limited due to genderdisparity, and societal power-dynamics (17). Previous studies have consistently reported an association between gender, socioeconomic gradients, religion and the prevalence of DM $(18,19)$. While some studies have even demonstrated that the risk factor burden for diabetes and other non-communicable diseases is greater among the higher socio-economic groups, mortality is lower, suggesting that wealthier groups have better access to treatments and healthcare, possibly through private insurance or through greater affordability of out-of-pocket health expenditures $(20,21)$.

This creates a set of concerns that are specific to women, and weaker sections of the population. Certain issues related to screening and management of vulnerable and high-risk groups includes patchy delivery of opportunistic screening, concerns about unmanageable workload, high burden on specialised care, and follow up of such patients. Therefore, it is pertinent that these vulnerable groups are fully aware of the available treatment options and their rights of access to services. Cost-effective interventions should be promoted to mitigate the rising burden of DM among these groups. In India, government has offered financial assistance through the Ayushman Bharat scheme, which is envisioned to curtail inequality, and key challenges like inadequate access to services, limited availability of medicines, and catastrophic out-of-pocket expenditure (OOPE). These challenges are also the bottlenecks to India's promise to accomplish universal health coverage (UHC) (22).

\section{Liberty}

Liberty supports the concept of patient autonomy and patient-centred care (PCC). PCC is defined as an approach to "providing care that is respectful of and responsive to individual patient preferences, needs, and values and ensuring that patient values guide all clinical decisions" (23).This involvement of patients in the informed decision-making process should be looked upon as the central principle of evidence based medicine for anyone who is living with a chronic disease. However, it is particularly suitable to DM. Simply, PCC involves crafting therapeutic plans that keep the preferences, needs and values of patients in mind. This allows as much flexibility, or "liberty," as possible (24). In diabetes management, although absolute liberty (in terms of permitting potentially harmful lifestyle choices) may not be possible, many different monitoring and treatment options are available that allow the patient to play an active role in therapy, based on the principles of informed decision making. Ultimately, it is patients who have to take a call pertaining to their lifestyle choices, and adherence to disease management protocols (including pharmacological and non-pharmacological interventions), that finally decide the disease outcome.
Also, denying liberty to debilitated patients living with DM, who lack the capacity to consent to their disease management protocols (eg patients with cognitive impairment), is a serious concern. In such cases, an amendment of the Mental Capacity Act, 2005, of England and Wales, also known as the Deprivation of Liberty Safeguards (DoLS), clarifies that such people may only be deprived of their liberty, if the protocols are made keeping in mind the best interest of such patient; and if there is no other alternative (25).

Responsible PCC reminds us that it is our duty to inform the person with diabetes about relevant aspects of his or her condition, so that appropriate decisions can be taken, to achieve optimal health.

Fraternity is another word used in the Indian Constitution. The constitution endeavours to promote fraternity amongst its citizens. This selfless ideal echoes in our modern concept of the diabetes team, peer-support, patient-provider interaction, and shared decision making. Keeping the person with diabetes as the centre of our focus, leaders in healthcare should facilitate incorporation of these constitutional goals in diabetes practice. Along with the right to euglycaemia, the process of achieving euglycaemia is equally important.

\section{Conclusions}

We have attempted to describe the core principles of the Indian constitution as they can be applied to ethical and updated medical care in patients living with DM. Together, these words remind us to practise accurate and appropriate diabetes care, delivered in a patient-centred manner. Justice, equality and liberty should be incorporated into diabetes care systems, and fraternity encouraged. Meanwhile, it will be interesting to watch how NCDs, particularly DM, will decide the fate of the development of our country in the coming decades. We should carefully tackle this crisis by taking inspiration from the past or present, in any form available. This will ensure that we achieve the dreams of our founding fathers through evolution of a comprehensive disease management model.

\section{References}

1. Davis S. The "social justice issue of our time": The challenge of noncommunicable diseases. Weforum.org. 2016 Sep 6 [cited 2020 May 13]. Available from: https://www.weforum.org/agenda/2016/09/thesocial-justice-issue-of-our-time-the-challenge-of-noncommunicable-diseases/.

2. World Health Organization. Global report on diabetes. Geneva: WHO; 2016 [cited 2020 May 13]. Available from: http://www.who.int/ about/licensing/copyright_form/index.html.

3. Tandon N, Anjana RM, Mohan V, Kaur T, Afshin A, Ong K, et al. The increasing burden of diabetes and variations among the states of India: the Global Burden of Disease Study 1990-2016. Lancet Global Health. 2018 Dec 1;6(12):e1352-62.

4. Tripathy JP, Thakur JS, Jeet G, Chawla S, Jain S, Pal A, et al. Prevalence and risk factors of diabetes in a large community-based study in North India: results from a STEPS survey in Punjab, India. Diabetol Metab Syndr. 2017 Dec;9(1):8.

5. Anderson RM, Heesterbeek $\mathrm{H}$, Klinkenberg D, Hollingsworth TD. How will country-based mitigation measures influence the course of the COVID-19 epidemic? Lancet. 2020 Mar 21;395(10228):931-4. 
6. Kalra S, Baruah MP, Sahay R. Salutogenesis in Type 2 diabetes care: a biopsychosocial perspective. Indian J Endocrinol Metab. 2018 Jan; 22(1):169.

7. Mathiharan K. The fundamental right to health care. Issues Med Ethics. 2003 Oct; 11:123.

8. Basu S, Sharma N. Under-recognised ethical dilemmas of diabetes care in resource-poor settings. Indian J Med Ethics. 2018 Oct-Dec; 3(4):324-6.

9. Williams CR, Buttfield B. Beyond individualised approaches to diabetes Type 2. Sociology Compass. 2016 Jun;10(6):491-505.

10. Gucciardi E, Vahabi M, Norris N, Del Monte JP, Farnum C. The intersection between food insecurity and diabetes: a review. Curr Nutr Rep. 2014 Dec 1;3(4):324-32.

11. Dixon LB, Winkleby MA, Radimer KL. Dietary intakes and serum nutrients differ between adults from food-insufficient and food sufficient families: Third National Health and Nutrition Examination Survey, 1988-1994. J Nutr. 2001;131(4):1232-46

12. Henning K. Addressing the gap in non-communicable disease data with technology and innovation,"[Internet]. Health Affairs Blog, [cited 2020 May 13]. Available from: https://www.healthaffairs.org/ do/10.1377/hblog20170921.062094/full/

13. Gupta I, Ranjan A. Public expenditure on Non-Communicable Diseases \& Injuries in India: A budget-based analysis. PLoS One 2019;14(9): e0222086.

14. Saligram N, Nugent R. Chronic diseases - The social justice issue of our time. Lancet. 2016 Mar 5;387(10022):943. Doi: 10.1016/S01406736(16)00569-9. Epub 2016 Mar 4.

15. European innovation partnership. Diabetes and cities: EIP partners work to develop Diabetes Prevention Score [Internet]. European innovation partnership on Active and Healthy Ageing. 2014 [cited 2020 May 13]. Available from: https://ec.europa.eu/eip/ageing/ news/diabetes-and-cities-eip-partners-work-develop-diabetes- prevention-score en

16. Horton R. Offline: Chronic diseases-the social justice issue of our time. Lancet. 2015 Dec;386(10011):2378.

17. Hannan C. Women, gender equality, and diabetes. Int J Gynecol Obstet. 2009 Mar;104(Supplement):S4-7.

18. Mutyambizi C, Booysen F, Stokes A, Pavlova M, Groot W. Lifestyle and socio-economic inequalities in diabetes prevalence in South Africa: A decomposition analysis. PLoS One. 2019 Jan 30; 14(1):e0211208.

19. Barua S, Saikia N. Explaining the religious disparity and associated risk factors of diabetes in Southern India, Eur J Public Health. 2018 May; 28. 10.1093/eurpub/cky047.042.

20. Corsi DJ, Subramanian SV. Association between socioeconomic status and self-reported diabetes in India: a cross-sectional multilevel analysis. BMJ Open. 2012 Jul 18;2(4):e000895. doi: 10.1136/ bmjopen-2012-000895.

21. Corsi DJ, Subramanian SV. Socioeconomic gradients and distribution of diabetes, hypertension, and obesity in India. JAMA Netw Open. 2019 Apr 5;2(4):e190411. Doi: 10.1001/jamanetworkopen. 2019.0411.

22. Verma M. Scope of management of Noncommunicable Diseases in India through Ayushman Bharat. JOSH-Diabetes. 2019 Dec;7(02):5860.

23. Bloom B. Crossing the quality chasm: a new health system for the 21st century. JAMA. 2002 Feb 6;287(5):646-7.

24. Kalra S. 'Life, Liberty and The Pursuit of Happiness' - The Unalienable Rights of Diabetes Care. Touch Endocrinology website. 2019 [cited 2020 May 13]. Available from:https://www.touchendocrinology.com/ insight/life-liberty-and-the-pursuit-of-happiness-the-unalienablerights-of-diabetes-care/

25. Aslam A, Rajbhandari SM. Deprivation of liberty to safeguard against recurrent ketoacidosis. Pract Diabetes. 2013 Mar;30(2):60-2.

\section{If you are looking for India's finest medical journal, then here it is.}

The National Medical Journal of India is a premier bi-monthly multi-disciplinary health sciences journal which publishes original research, reviews, and other articles relevant to the practice of medicine in India. The journal aims to instruct, inform, entertain and provide a forum for the discussion of social, economic and political health issues. It is included in the Index Medicus, (MEDLINE), Excerpta Medica (EmBase), BIOSIS, Current Contents/Clinical Medicine and Science Citation Index.

\begin{tabular}{|c|c|c|c|c|}
\hline \multicolumn{5}{|c|}{ SUBSCRIPTIONS } \\
\hline & One year & Two years & Three years & Five years \\
\hline India & Rs. 800 & Rs. 1500 & Rs. 2200 & Rs. 3600 \\
\hline Overseas & US $\$ 100$ & US \$180 & US \$270 & US \$450 \\
\hline
\end{tabular}

Personal subscriptions paid from personal funds are available at $50 \%$ discounted rates

Bank draft/cheques should be made in favour of The National Medical Journal of India. Journals can be sent by registered post on request at an added cost of Rs 90 per annum. Requests to be made at the time of subscribing.

Tel:91-11-26588802 Fax:91-11-26588663 E-mail:nmji@nmji.in Website:www.nmji.in

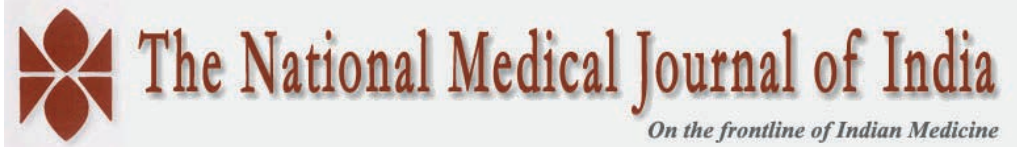

